\title{
Study on the influencing factors and rules of the phased evacuation of building
}

\author{
Li Yang ${ }^{1}$, Daoliang Zhao ${ }^{1 *}$, Meiting Zeng ${ }^{1}$,Yun Lu ${ }^{1}$, Lei Shi ${ }^{1}$, Hu Zhou ${ }^{1}$ \\ ${ }^{1}$ College of Urban Construction and Safety Engineering, Shanghai Institute of Technology, Shanghai, China
}

\begin{abstract}
The whole evacuation of building fire personnel is always a research hotspot. However, in special occasions and conditions, evacuation to the first safety zone as soon as possible is a powerful way to ensure the safety of people's lives, that is, the study of the phased evacuation of building is also of great practical significance. In this paper, the BuildingEXODUS software was used to establish the evacuation model, which mainly analyzed the influence and law of two physical factors on the evacuation of people from the room to the corridor: personnel density, personnel distribution in the corridor. In addition, MassMotion software was used to simulate and compare some conclusions under the same evacuation scene, aiming to study and compare the similarities and differences of the influence of the same physical factors on the phased evacuation of building under different simulation mechanisms.
\end{abstract}

\section{Introduction}

When an emergency occurs in a specific building, organizing personnel to flee from the building in a short time can minimize or avoid casualties, that is, orderly evacuation is an important guarantee for public safety ${ }^{[1]}$. Xue et al. ${ }^{[2]}$ mainly discussed the impact of the width and position of the exit of the room and the width of the corridor on the whole evacuation efficiency. Liao et al. ${ }^{[3]}$ first conducted a questionnaire survey, and then simulated the evacuation of a teaching building through Anylogic, it is obtained that the evacuation efficiency of the whole teaching building has been improved under the additional sign.

The overall evacuation of construction personnel is important in the event of an accident, but in some special circumstances, for those who are on the scene of the first accident, it is more important to evacuate from dangerous places to relatively safe areas in the shortest possible time.

There are many kinds of fire hazard sources inside the hospital, so there are certain fire safety hazards, however, apart from medical staff and family members, there are also patients with mobility difficulties, which leads to great difficulty in evacuation ${ }^{[4,5,6]}$. A common scene in a hospital is a doctor in an outpatient department in a room to diagnose a patient, while people outside the room wait in the hallway for a call. If a fire breaks out in a room, evacuating the entire building can cripple the health care system, the most unfavorable situation is to cause a certain number of people panic, and patients already with mobility difficulties may be trampled or pushed by the crowded crowd, which will aggravate their condition, obviously with disastrous consequences. At this time, it is crucial for people in the room to evacuate quickly from the room to the corridor. $\mathrm{Li}$ et al. ${ }^{[7]}$ believed that the temporary safety area obtained from the transformation of the evacuation walkway could solve the problem of people escaping due to insufficient evacuation width of the building when meeting certain functional requirements. Tian et al. ${ }^{[8]}$ took the evacuation walkway as a quasi-safety zone to solve the problem that the fire protection zone in the underground cultural heritage storehouse of a museum exceeds the standard, on the premise that the fire parameters of the walkway do not pose a danger to the safety of evacuators, finally, based on the simulation, it was concluded that the performance-based design of the quasi-safety zone was feasible. Moreover, it is pointed out in 《the Performance-based Design of Building Fire Prevention》 ${ }^{[9]}$ that the evacuation walkway is called the first safety zone in the evacuation design. The quasi-safe area is a relatively safe area that meets certain performance indicators ${ }^{[10]}$, the first safety zone is the area with certain safety performance that evacuees first arrive at, therefore, under reasonable structural conditions, the evacuation walkway can become a safer area compared with the danger area during evacuation.

The first stage of the evacuation route in the building is from the burning room to the door of the room ${ }^{[9]}$, then arrive at the evacuation walkway (corridor), evacuation efficiency and evacuation time in this stage may be related to personnel density, personnel distribution and other factors. In this paper, BuildingEXODUS software was used to simulate and analyze the influence of these physical factors on the first-stage evacuation process, and MassMotion software was used to simulate and compare, in order to obtain the behavior rule of the first-stage evacuation of building personnel. 


\section{Software Comparison and Parame- ter Introduction}

In BuildingEXODUS, the attribute of an individual searching for the destination during evacuation is set as "Nearest door", there is no specific direction bias during the movement.

In MassMotion, the attribute of an individual searching for the destination during evacuation is set as "lowest cost". In addition, evacuees are set to have "Direction Bias", that is, the preferred direction to avoid oncoming traffic, and set to "Right strong".

In this paper, BuildingEXODUS software was used to simulate and study the impact of physical factors on the efficiency of room staff evacuation to the corridor. It is mainly analyzed according to the simulated scenario and the last entry data of each internal exit.

Last entry means the last usage time of the internal exit. In this paper, the time of the last person in the room arriving at the internal exit is considered according to the last entry and the simulated scenario, that is, how long it takes the most dangerous people to escape from the room. The following are the arrival time of the final personnel at internal exits.

\section{Evacuation Simulation Model}

The evacuation model in this article is created based on the geometric plane shown in Fig.1, the effective size is as follows: the corridor is $13 \mathrm{~m} \times 2.5 \mathrm{~m}$, the room is $4 \mathrm{~m} \times 2 \mathrm{~m}$, the external exit on the left is $1.5 \mathrm{~m}$, the external exit on the right is $2.5 \mathrm{~m}$ and the internal exit is $1 \mathrm{~m}$. The maximum capacity of the corridor is 130 people, the maximum capacity of each room is 32 people, the evacuation speed was set as $1.2 \mathrm{~m} / \mathrm{s} \sim 1.5 \mathrm{~m} / \mathrm{s}$, and the response time was $0 \sim 30$ s. In order to reduce the difference of the data generated by the random distribution of personnel, each set of simulations for the full text was run five times.
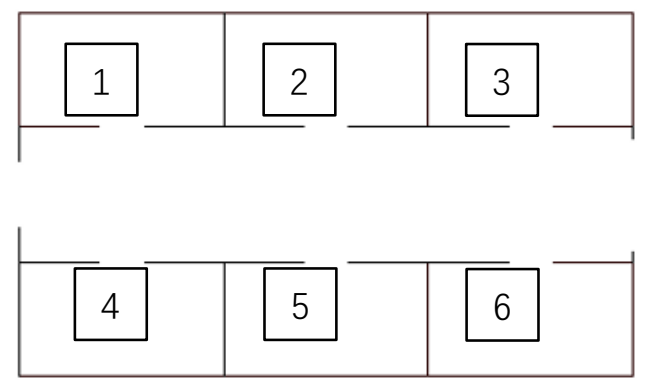

Fig1. Geometric plane of evacuation model

\section{Simulation Results Analysis}

\subsection{The Influence of Personnel Density on the Evacuation of Room Personnel}

The simulation in this part mainly considers the impact of personnel density on the evacuation of room personnel. Setting the number of people in each room at five initial moments in BuildingEXODUS, that is, 6, 12, 18, 24 and 30. According to the specific number of people in each room, increase the number of people in the corridor successively from 0-120, 20 people were increased each time, and finally five groups of simulations were carried out. The origin software was used to fit the quadratic polynomial of the arrival time of the final personnel at internal exits, as shown in Fig.2.

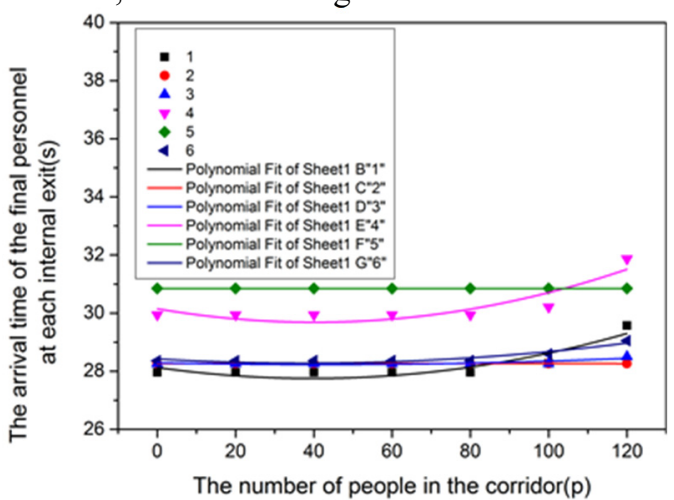

a. the number of people in each room at the initial time is 6

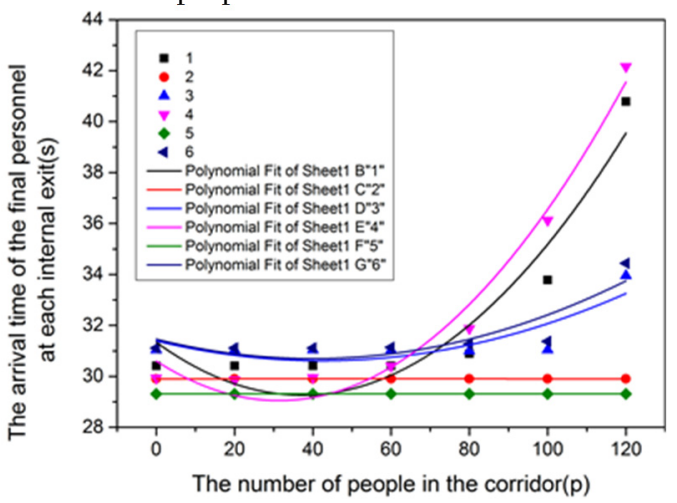

b. the number of people in each room at the initial time is 12

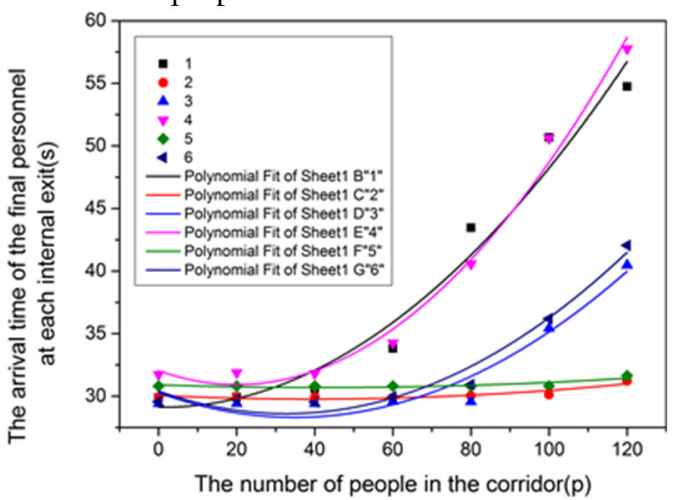

c. the number of people in each room at the initial time is 18

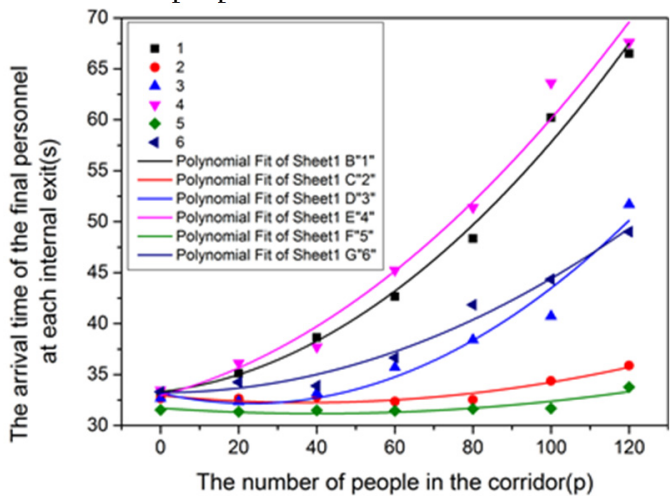

$\mathrm{d}$. the number of people in each room at the initial time is 24 


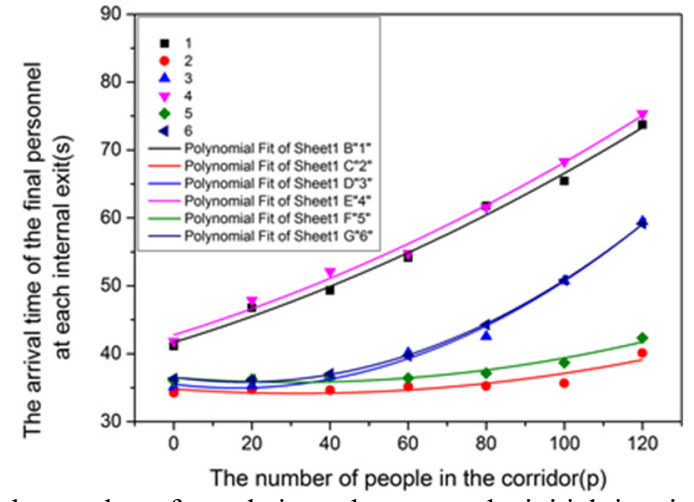

e. the number of people in each room at the initial time is 30 Fig2. The effect of change in personnel density on the arrival time of the final personnel at internal exits

It is easy to see from Fig. 2 that all internal exits fall into three categories. The first type is No. 1 and 4, the second type is No. 2 and No. 5, and the third type is No. 3 and No. 6.

With the increase of the personnel density of rooms, the influence of changing the personnel density of the corridor on the arrival time of the final personnel at internal exits of the first type and the third type are more and more obvious, $b 、 c 、 d$ and e are shown in Fig.2. In addition, the arrival time of the final personnel between the first, second and third types of internal exits gradually starts to produce a certain gap under a smaller personnel density of the corridor, because the number of people in rooms increases and the width of the external exits on the left and right is different. When the personnel density of rooms is high, as shown in Fig.2e, the arrival time of the final personnel at internal exits of the first type increases almost linearly.

On the premise that the personnel density of rooms is low, if the personnel density of the corridor is not high, the arrival time of the final personnel at internal exits of these three types is not different, if the personnel density of the corridor is high, the general rule of the arrival time of the final personnel at internal exits is $1,4>3 、 6>2 、 5$, as shown in Fig.2b. On the contrary, when the personnel density of rooms is relatively high, change the personnel density of the corridor, and the arrival time of the final personnel generally presents such a rule. For individuals looking for destination in BuildingEXODUS is " Nearest door ", finally the difference in the number of people who pass through the two external exits is small, but because the width of the external exit on the left is smaller than that of the external exit on the right, when the evacuees gradually approach external exits, the left side of the corridor is more likely to form congestion, resulting in the delay of the arrival time of the final personnel at internal exits of the first type. In addition, with the evacuation to both sides of the corridor, the number of people in the middle gradually decreases, so that the change of personnel density in the corridor has little impact on the arrival time of the final personnel at the second type of internal exits.

According to the simulation of BuildingEXODUS software, when the personnel density of rooms is relatively high, change the personnel density of the corridor, and the arrival time of the final personnel at each internal exit is generally $1 、 4>3 、 6>2 、 5$. At the initial moment, 30 people in the room and 100 people in the corridor were set, and the simulation results were compared under the same scene with MassMotion software, as shown in Fig.3.

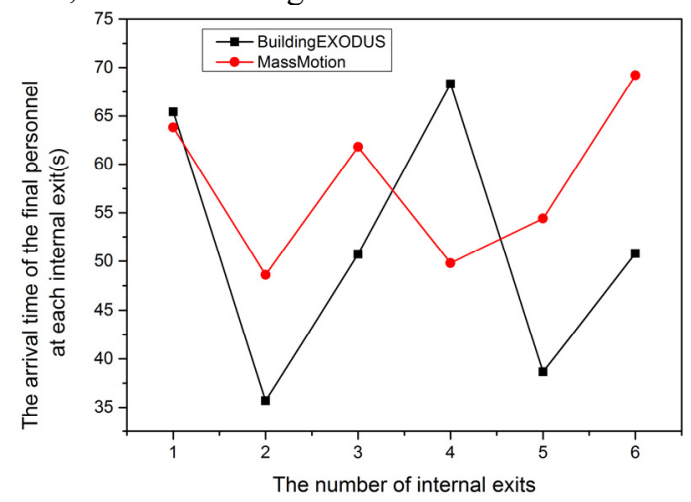

Fig3. The final arrival time of each internal exit with 30 people in the room at the initial time and 100 people in the corridor

In this part of the simulation, the "lowest cost" attribute of individual searching for the destination in the evacuation process in MassMotion improves the overall evacuation efficiency of the building. Facing the external exits with different widths on the left and right, the number of people passing through the external exits varies greatly, while the difference in the final passing time is small.

As can be seen from Fig.3, when the personnel density of both the room and corridor is high, what is different from the simulated result of BuildingEXODUS is that the arrival time of the final personnel at the internal exit 1 and 4 near the external exit on the left is greatly different and does not belong to the same category, so are those at the internal exit 3 and 6 near the external exit on the right, while the arrival time of the final personnel at the internal exits of 2 and 4 is similar, and the difference of 1 and 3 is also small. In MassMotion simulation, there is a big difference in the number of people passing through external exits on the left and right, making the total number of people crowded near the internal exit 1 and 4 less, and the number near the internal exit 3 and 6 more. However, because the direction bias of individuals is right strong, when people are evacuated in the corridor and facing the stream of people at two internal exits at the same time, they tend to be on the right side of the evacuation direction of the corridor, and the density near the internal exit 1 is also high. At the same time, the direction bias of individuals is also the reason why two internal exits located in the same position of the corridor do not belong to the same kind.

\subsection{The Impact of the Personnel Distribution in the Corridor on the Evacuation of Room Personnel}

The simulation in this part mainly analyzes the impact of the personnel distribution in the corridor on the evacuation of the room. When using BuildingEXODUS to simulate, set the number of people in the corridor at 80 and set three initial personnel distribution states of the corridor, that is, random, middle and on both sides, as shown in Fig.4. 


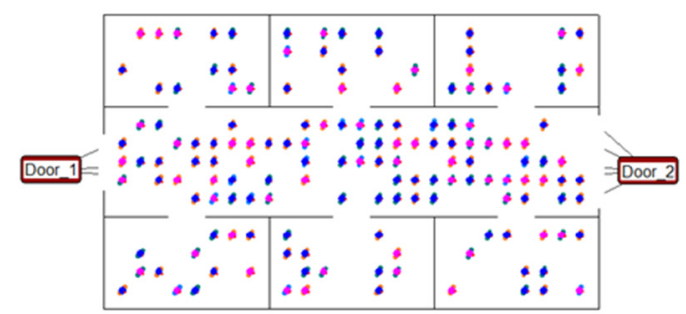

a. The corridor personnel were randomly distribute

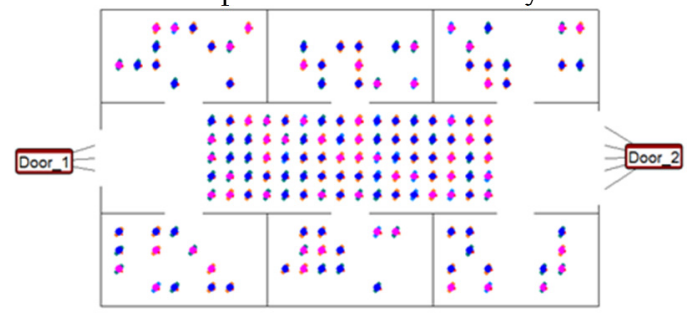

b. The corridor personnel were distributed in the middle

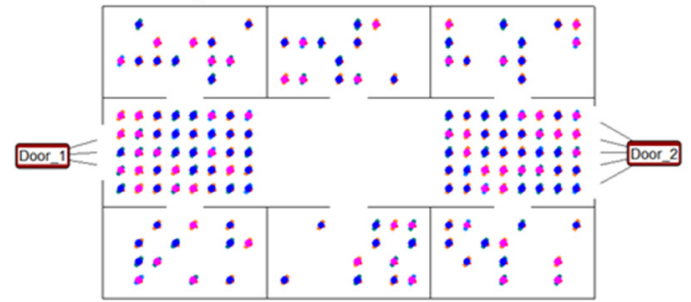

c. The corridor personnel were distributed on both sides

Fig4. Three types of corridor personnel distribution of 12 people in the room at the initial moment

The origin software was used to fit the quadratic polynomial of the arrival time of the final personnel at internal exits, as shown in Fig.5.

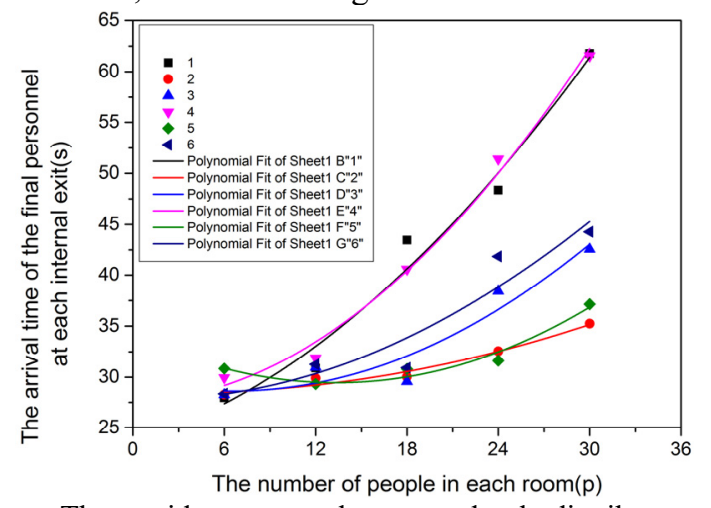

a The corridor personnel were randomly distribute

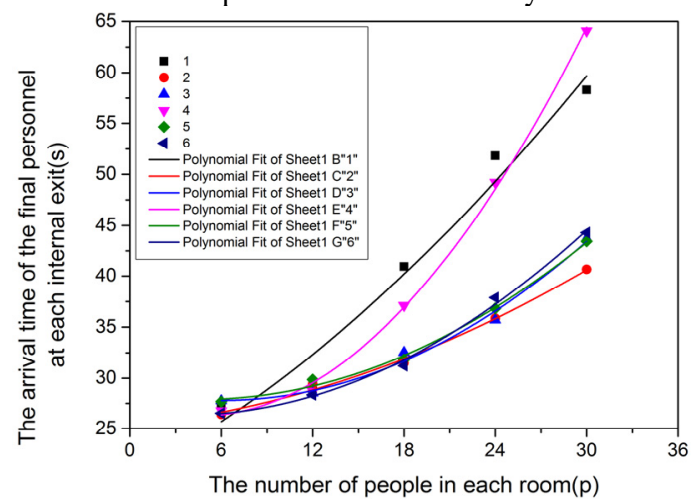

$\mathrm{b}$ The corridor personnel were distributed in the middle

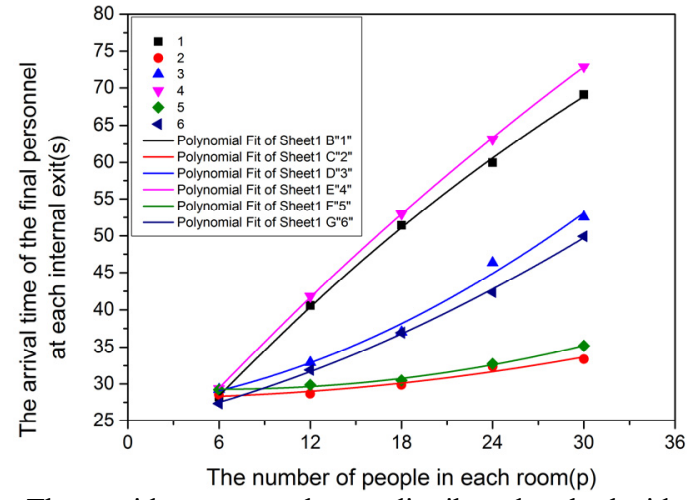

$c$ The corridor personnel were distributed on both sides Fig5. Influence of different corridor personnel distribution on the final arrival time of internal exits

As in the BuildingEXODUS simulation in Section 4.1, all internal exits are divided into three categories: the first type is No. 1 and No. 4, the second type is No. 2 and No. 5 and the third type is No. 3 and No. 6.

It can be seen from Fig. 5 that, with the increase of the personnel density of rooms, the difference between the arrival time of the final personnel at internal exits of the first type in the random distribution of corridor personnel and the middle distribution is relatively small, which is about $3.5 \mathrm{~s}$ at most. When people in the corridor are distributed on both sides, on the premise of low density of people in the room, there is a small difference between the arrival time of the final personnel at internal exits of the first type and the two situations above. For example, when the number of people in each room is 6 , the difference is small because the number of people is low. The personnel density of the room gradually increases, and the arrival time of the final personnel at internal exits of the first type is higher when the corridor personnel are distributed on both sides. In this case, the people in the vicinity are in a dense state at the initial moment, and the number of people in each room is relatively large, so it is not easy for the people in the room to evacuate quickly, thus increasing the arrival time of the final personnel. In addition, the average evacuation efficiency of rooms near the external exit on the left under the previous two distributions increased by about $27.6 \%$ at the highest level compared with that of the distribution on both sides. The situation of the third type of internal exits is similar. The difference between the arrival time of the final personnel in the random distribution of corridor personnel and the middle distribution is small, the most is about $4 \mathrm{~s}$. Moreover, the average evacuation efficiency of rooms near the external exit on the right under these two distributions increases by about $20.08 \%$ at the highest level compared with that of the distribution on both sides.

It is also easy to conclude that, as the personnel density of the room increases, the arrival time of the final personnel at internal exits of the second type is similar in the case of the random distribution of corridor personnel and the distribution on both sides, with a maximum difference of about $2 \mathrm{~s}$. When the corridor personnel are distributed in the middle, if the personnel density of the room is low, the difference between the arrival time of the final personnel and that of the previous two distributions is small, such as the number of people in each room is 18 . As can be seen from Fig.6, the middle distribution of 
corridor personnel makes the initial time near the internal exits of the second type more dense, but people around the 20 s are evacuated and separated, which reduces the density. If the number of people in the room is not particularly large, they will evacuate to the corridor more quickly. If the corridor personnel are distributed in the middle, the arrival time of the final personnel is greater in the case of a larger number of people in the room than in the case of the other two distributions, for example, the number of people in each room is 30 . Although people distributed in the middle of the corridor have not been evacuated to both sides for a long time, there are enough people in the room. In addition, the impact of dense personnel in the corridor before evacuation and separation leads to the growth of the arrival time of the final personnel at internal exits of the second type. The average evacuation efficiency of the rooms in the middle under the random distribution of corridor personnel and the distribution on both sides increased by about $16.76 \%$ at the highest level compared with the middle distribution.

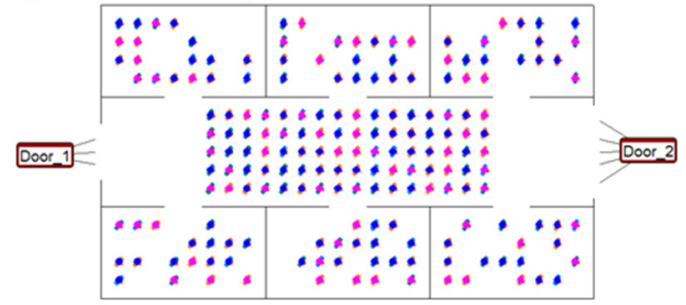

a. When the simulation time was $0 \mathrm{~s}$

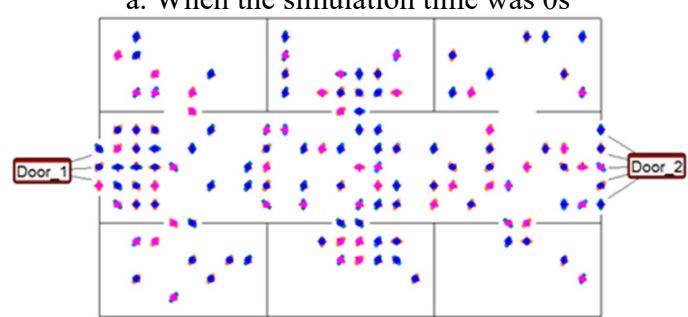

b. When the simulation time was $20 \mathrm{~s}$

Fig6. A simulation scenario with 18 people in the room at the initial time and the people in the corridor distributed in the middle

According to the results of BuildingEXODUS, the difference of the arrival time of the final personnel at internal exits of the first type $(1,4)$ is small in the case that the corridor personnel are randomly distributed and in the middle. In addition, as the personnel density of the room increases gradually, the arrival time of final personnel is higher when the corridor personnel are distributed on both sides than in the previous two distribution situations. The third type of internal exits $(3,6)$ have similar situation. Examples are shown in Fig.7. MassMotion software was used to simulate in the same scene, and the results are shown in Fig.8.

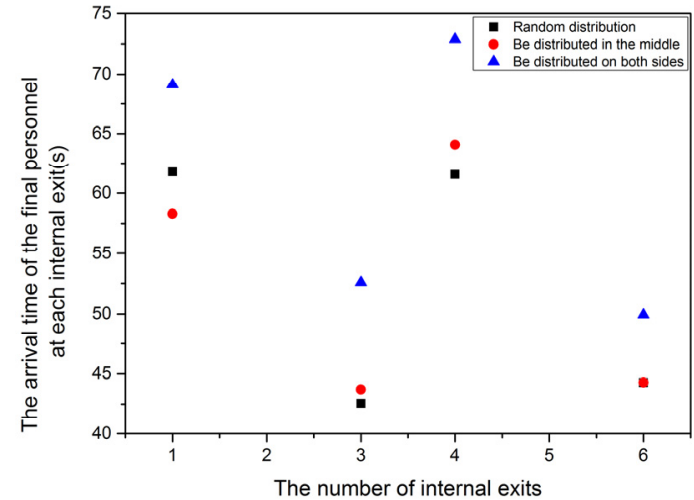

Fig7. Simulation results of BuildingEXODUS for 30 people in the room at the initial time

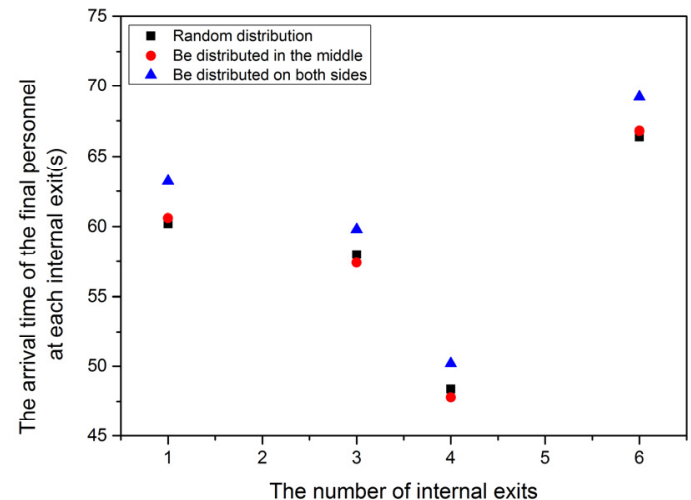

Fig8. Simulation results of MassMotion for 30 people in the room at the initial time

It is the same as that obtained in MassMotion simulation in Section 4.1, because of the problem of direction bias, the No.1 and No.4 internal exit close to the external exit on the left in MassMotion do not belong to the same category, and the same is true for No.3 and No.6 internal exit.

It can be seen from Fig. 8 that when the density of people in the room is high, the difference in the arrival time of the final personnel at the No.1 internal exit is small in the three distribution forms of corridor personnel, and the same is true for No.3、No.4、No.6 internal exit. However, it is also easy to find that the arrival time of the final personnel at four internal exits is slightly higher than that under the random and middle distribution when the corridor personnel are distributed on both sides. For example, the reason of No.1 internal exit is the same as that in BuildingEXODUS, because the corridor personnel are distributed on both sides at the initial moment, the people near the internal exit will have a long time of congestion, but in MassMotion, there are fewer people in the final moment, which has a small impact, so the difference between the three distributions is not big.

\section{Conclusion}

1) BuildingEXODUS was used to simulate the initial personnel density of the room and corridor, which has a great influence on the evacuation efficiency of rooms near the two external exits, but a small influence on the evacuation efficiency of the middle rooms. And the change in the arrival time of the final personnel at two internal exits near the external exit on the left belongs to 
the same category, that of two middle internal exits also belongs to the same kind, the same is true for two internal exits near the external exit on the right. In MassMotion, when the personnel density of both the room and corridor is high, the difference of the arrival time of the final personnel at two internal exits near the external exit on the left is larger, that of two internal exits close to the external exit on the right is also larger, and that of two middle internal exits is not similar.

2) In the simulation of BuildingEXODUS, the initial personnel distribution in the corridor will affect the phased evacuation efficiency of room personnel. The difference of evacuation time of people in rooms near the external exits is smaller in the random distribution of corridor personnel and the middle distribution, while the difference of evacuation time of people in middle rooms is smaller in the random distribution of corridor personnel and the distribution on both sides. In MassMotion, the evacuation time of each room near external exits varies little among the three distribution forms of corridor personnel.

\section{Acknowledgments}

Project of Shanghai Natural Science Foundation (15ZR1440900) ;

Science and Technology talent Development Fund for young and middle-aged teachers of Shanghai Institute of Technology (ZQ2018-21)

\section{References}

1. W.H. Wang, S.X. Wu, J. Cheng. Mathematical Model of Evacuation Program in a Building[J]. Journal of Wuhan University of Technology, 32(11):155$158+162(2010)$.

2. P. Xue, J.W. Zhou, K.Z. Bai, L.J. Kong, M.R. Liu. Cellular Automaton Model of Occupant Evacuation from Multi-room in Corridor[J]. Journal of Guangxi Normal University(Natural Science Edition), 27(03):1-4(2009).

3. H.M. Liao, X.J. Luo, H. Su. Research on cognitive coping regularity of evacuation signs in fire of teaching building $[\mathrm{J}]$. Journal of Safety Science and Technology, 15(08):131-136(2019).

4. F. Yang, J. Chen. On the Risk Assessment of Fire Hazards in Hospitals[J]. The Science Education Article Collects, (16):190-192(2015).

5. S.B. Li. Discussion on hospital fire safety management and emergency[J].Jiangsu Health System Management, (1):133-134(2014).

6. H.B. Liang. Fire safety management and emergency of large hospital buildings[J].Modern Preventive Medicine, 34(7):1327-1327(2007).

7. F. Li, Y. Zhang. Solutions on the lack of evacuation width in existing building $[\mathrm{J}]$. Fire Science and Technology, 33(08):894-896(2014).

8. Y.H. Tian, G. Li. Fire performance-based design of a museum[J]. Fire Science and Technology, 32(4):391393(2013).
9. F. Liu, S.J. Liao. Safe evacuation of personnel. In: H.M. Chen, H.G. Zhou(Eds.), the Performance-based Design of Building Fire Prevention[M].CHONGQING UNIVERSITY PRESS, Chongqing, 147-151(2007).

10. G.W. Zhang, G.Q. Zhu, W.H. Wu, L.L. Huang. Judging method of "quasi safe area" of building based on performance-based fire protection design[J].China Safety Science Journal, 21(04):60-65(2011). 